\title{
Mapping and elucidating the function of modified bases in DNA
}

Eun-Ang Raiber, Robyn Hardisty, Pieter van Delft and Shankar Balasubramanian

Nature Reviews Chemistry 1, 0069 (2017)

In figure 2 of this article, an incorrect structure for 5 -formyluracil was published in the online version, and a definition of the R group was omitted from the legend in both the online and PDF versions. These errors have now been corrected in their respective versions. The corrected figure and legend are shown below. We apologize to readers for any confusion caused.

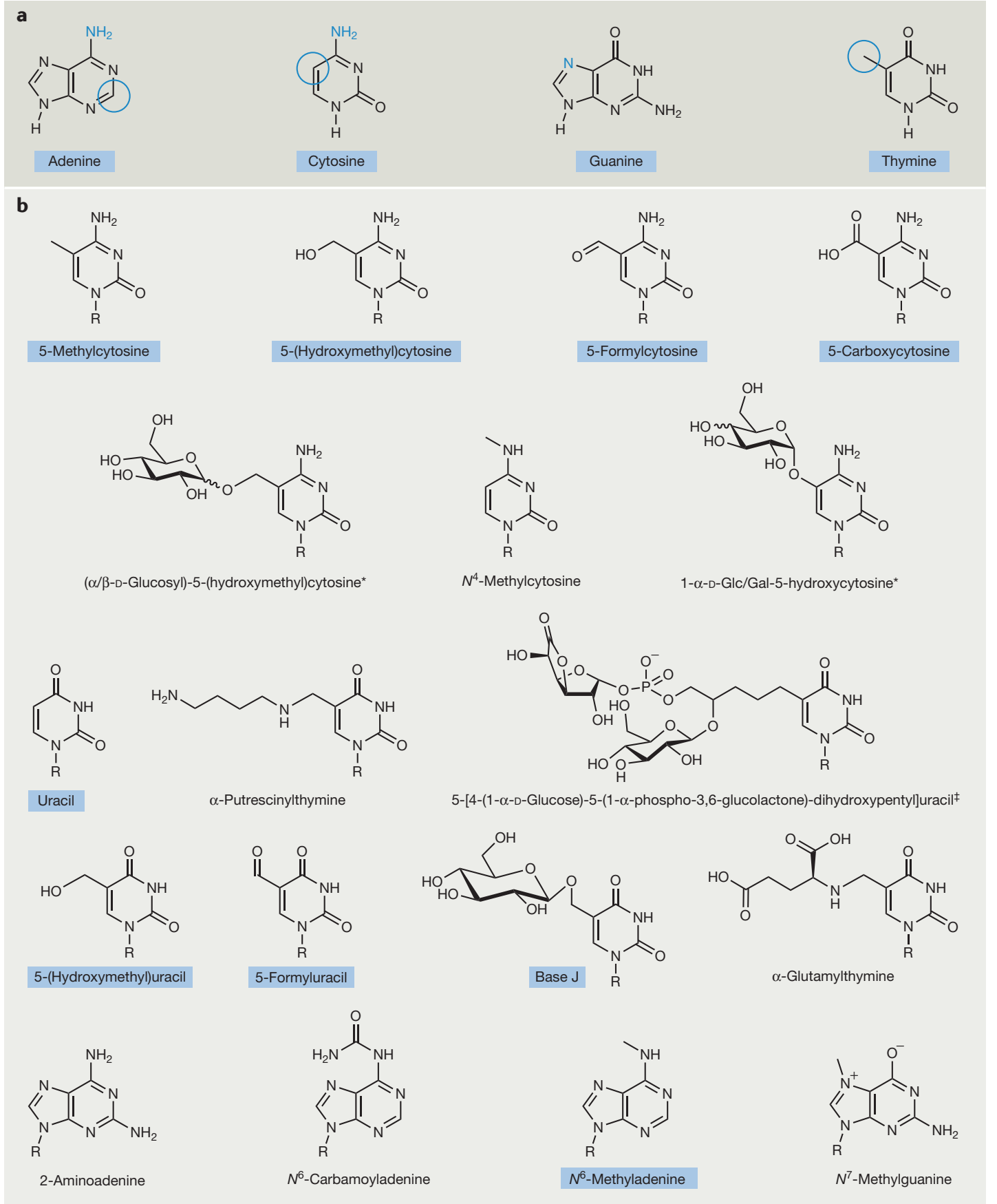

Figure 2 | Overview of modified DNA bases. a | The four canonical bases are depicted, with the sites of known modifications highlighted. $\mathbf{b}$ |Chemical space of the modified DNA bases that have been reported in the literature. Structures with names in boxes are modifications that have been identified in eukaryotic genomes and are discussed in the scope of this Review; other modifications have been reviewed elsewhere ${ }^{5}$. For DNA, R indicates 2'-deoxy-D-ribose. *More-complex branching of mixtures with up to three furanose residues (Glc and Gal) have also been reported.

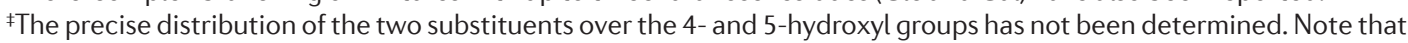
$\mathrm{N}^{6}$-methyladenosine is shown in its energetically preferred cis conformation. 